\title{
Identificação e controle de biótipos resistentes de Digitaria insularis (L.) Fedde ao glyphosate ${ }^{1}$
}

\section{Identification and control of Digitaria insularis biotypes resistant to the glyphosate}

\author{
Leandro Rissieri Licorini ${ }^{2}$; Marco Antonio Gandolfo ${ }^{3}$; Maria Aparecida Sorace $^{3}$; Robinson \\ Osipe $^{3}$; Conceição Aparecida Cossa ${ }^{3}$; Jethro Barros Osipe ${ }^{3}$
}

\begin{abstract}
Resumo - Em áreas de plantio direto, algumas espécies invasoras têm apresentado potencial para desenvolver resistência ao herbicida glyphosate. Herbicidas inibidores da enzima ACCase podem constituir uma alternativa de controle de biótipos de Digitaria insularis (L.) Fedde resistentes ao glyphosate. Os objetivos deste trabalho foram identificar a ocorrência de resistência em biótipos de $D$. insularis (L.) Fedde ao glyphosate em áreas de plantio direto de municípios do Estado do Paraná e encontrar as doses de clethodim adequadas para seu controle. Foram conduzidos dois experimentos em casa de vegetação no município de Bandeirantes-PR, sendo o primeiro com o herbicida glyphosate e o segundo, com o herbicida clethodim. Sementes de plantas de $D$. insularis (L.) Fedde foram coletadas em cinco locais diferentes e curvas de dose-resposta foram obtidas para a verificação da resistência. As doses testadas foram equivalentes a 0;0,5; 1; 2; 3 e 4 vezes as doses recomendadas dos herbicidas. No momento da aplicação, as plantas apresentavam de 15 a $20 \mathrm{~cm}$ de altura. Os resultados mostraram que os biótipos de $D$. insularis (L.) Fedde oriundos das cidades do Paraná são resistentes ao herbicida glyphosate. Além disso, verificou-se tolerância diferenciada de tais biótipos ao herbicida clethodim.
\end{abstract}

Palavras-chave: capim-amargoso; clethodim; controle

Abstract - Some invasive species has shown potential to develop resistance to glyphosate herbicide in no-till areas. Herbicides inhibitors of ACCase enzyme may be an alternative control to Digitaria insularis (L.) Fedde. The objectives of this study were to identify the occurrence of resistance in $D$. insularis (L.) Fedde to glyphosate in tillage areas of the State of Paraná, and find the appropriate clethodim doses for its control. Were conducted two experiments in a greenhouse in Bandeirantes-PR, where the first was the experiment conducted with the glyphosate herbicide and the second with clethodim herbicide. Seeds of D. insularis (L.) Fedde plants were collected from five different places, and dose response curves were obtained for resistance verification. The tested doses were equivalent to $0 ; 0.5 ; 1 ; 2 ; 3$ and 4 times the recommended dose of herbicide. The plants had 15 to $20 \mathrm{~cm}$ high when the herbicides were sprayed. The results showed that $D$. insularis (L.) Fedde biotypes from Paraná cities are resistant to glyphosate herbicide. Furthermore, it was found differentiated tolerance to the clethodim herbicide in the biotypes.

Keywords: sourgrass; clethodim; control

\footnotetext{
${ }^{1}$ Received for publication on 20/07/2015 and approved on 22/10/2015.

${ }^{2}$ Engenheiro Agrônomo de Desenvolvimento da Bayer Cropscience, Rua José Pedro, 281, Bandeirantes - PR, CEP: 86360-000, e-mail: leandrolicorini@yahoo.com.br

${ }^{3}$ Professor Doutor da Universidade Estadual do Norte do Paraná Campus Luiz Meneghel (UENP-CLM), Centro de Ciências Agrárias, Bandeirantes - PR; Br 369, Km 54, CP 261 - CEP: 86360-000
} 


\section{Introdução}

O sistema de semeadura direta tem se intensificado nos últimos anos e a sua viabilização está relacionada diretamente com a disponibilidade e utilização de herbicidas eficazes para o manejo das plantas daninhas e dessecação das culturas.

O glyphosate é o principal herbicida utilizado na atualidade no manejo das comunidades infetantes, porém a intensificação do seu uso pode contribuir significativamente para a seleção de biótipos de espécies de plantas daninhas naturalmente resistentes pré-existentes nas populações (Koger e Reddy, 2005).

$\mathrm{O}$ aumento de plantas daninhas resistentes aos herbicidas é problema crescente em muitos países. Entre as espécies comprovadamente resistentes ao glyphosate está o capim-amargoso (Digitaria insularis (L.) Fedde). Os mecanismos que conferem resistência em $D$. insularis (L.) Fedde estão relacionados com a absorção mais lenta e à metabolização mais rápida do glyphosate, glioxilato e sarcosina, além da translocação do herbicida ser muito menor em plantas resistentes do que em suscetíveis, mesmo com 3-4 folhas (Carvalho et al., 2011).

As plantas de D. insularis (L.) Fedde se destacam pelas características de agressividade que proporcionam a sobrevivência em ambientes com vários tipos e intensidades de limitações ao seu crescimento e ao desenvolvimento (Brighenti e Oliveira, 2011). Dentre as características, destaca-se a formação de rizomas, que surge normalmente após 35-40 dias da emergência da planta (Machado et al., 2006). Isso pode dificultar a translocação de herbicidas, permitindo rápida rebrota da parte aérea (Machado et al., 2008).

Alternativa de controle das plantas daninhas resistentes ao glyphosate (inibidor de EPSPs), como a aplicação de clethodim (inibidor da ACCase) tem sido recomendada para o controle em pós-emergência de gramíneas anuais e perenes com alta eficácia (Spader et al., 2008). Barros et al. (2014) verificaram que herbicidas como quizalofop, haloxyfop, sethoxydim e clethodim são ótimas opções de controle para o capim-amargoso.

O objetivo deste trabalho foi identificar a ocorrência de resistência em $D$. insularis (L.) Fedde ao glyphosate em áreas de plantio direto de municípios do Estado do Paraná, além de encontrar as doses de clethodim adequadas para seu controle.

\section{Material e Métodos}

Dois experimentos foram realizados em casa de vegetação no município de Bandeirantes-PR com plantas de capimamargoso (D. insularis (L.) Fedde) no ano de 2013. No primeiro, o herbicida utilizado foi o glyphosate, e no segundo, o clethodim.

Para isso, foram coletadas sementes de capim-amargoso em seis diferentes locais, sendo cinco com suspeita de resistência ao herbicida glyphosate e um, em área na qual não se aplicou este herbicida nos últimos cinco anos (área suscetível).

Para o primeiro experimento (herbicida glyphosate), o delineamento experimental adotado foi o inteiramente casualizado, em esquema fatorial ( 5 x 6), sendo cinco locais de coleta de sementes e seis dose do herbicida, com cinco repetições. A unidade experimental foi representada por vaso de $1 \mathrm{~L}$ com uma planta de capim-amargoso. As sementes de plantas de $D$. insularis com suspeita de resistência foram coletadas em áreas dos municípios de: Cascavel (Área 1), Palotina (Área 2), Santa Mariana (Área 3), Maringá (Área 4) e Leópolis (Área 5 suscetível) no Estado do Paraná. Para o segundo experimento com o herbicida clethodim, apenas os locais com suspeita de resistência ao glyphosate foram testados, visto que o objetivo era determinar as doses necessárias de controle para estas áreas. Assim, o delineamento experimental adotado para o segundo experimento foi o inteiramente casualizado, em esquema fatorial ( $4 \times 6$ ), sendo quatro locais de coleta de sementes e seis dose do herbicida, com cinco repetições. 
As doses dos herbicidas foram equivalentes a $0 ; 0,5 ; 1 ; 2 ; 3$ e 4 vezes as doses recomendadas dos herbicidas. Para o glyphosate utilizou-se $0 ; 480 ; 960 ; 1920 ; 2880$ e 3840 g e.a. $\mathrm{ha}^{-1}$, e para o clethodim, $0 ; 48 ; 96 ; 192 ; 288$ e 384 g i.a. ha ${ }^{-1}$. No segundo experimento, todos os tratamentos receberam óleo mineral a $0,5 \%$ $\mathrm{v} / \mathrm{v}$.

A aplicação dos herbicidas foi realizada quando as plantas daninhas apresentavam 15-20 $\mathrm{cm}$ de altura, com dois perfilhos. Para a aplicação dos herbicidas, foi utilizado pulverizador costal pressurizado a $\mathrm{CO} 2$, com pressão constante de trabalho de $200 \mathrm{kPa}$ (29 PSI), equipado com lança contendo 4 pontas de pulverização do tipo jato plano modelo XR110 02, o que resultou numa taxa de aplicação de $200 \mathrm{~L} \mathrm{ha}^{-1}$.

As condições climáticas no momento da aplicação dos tratamentos foram: velocidade do vento inferior a 0,77 m.s. ${ }^{-1}$, temperatura de $26^{\circ} \mathrm{C}$ e umidade relativa do ar de $75 \%$.

As avaliações de controle foram realizadas utilizando escala visual de 0 a $100 \%$, em que $0 \%$ representa nenhum controle ou ausência de controle e $100 \%$, controle excelente das plantas daninhas presentes aos 28 dias após a aplicação (D.A.A.).

Os resultados foram submetidos à análise estatística inicialmente por meio da aplicação do Teste $\mathrm{F}$ na análise de variância para identificação da diferença de resposta dos biótipos aos tratamentos com os herbicidas e posteriormente à análise de regressão nãolinear, utilizando o modelo proposto por Streibig, (1988):

$$
\mathrm{Y}=\frac{a}{\left[1+\left(\frac{x}{b}\right)^{c}\right]}
$$

Em que:

$\mathrm{Y}=$ controle percentual;

$x=$ dose do herbicida (g i.a. ou e.a.ha ${ }^{-1}$ ); equação:

$a, b$ e $c=$ parâmetros estimados da

$a=$ amplitude existente entre o ponto máximo e o ponto mínimo da variável; $b=$ dose que proporciona $50 \%$ de resposta da variável;

$c=$ declividade da curva ao redor de $b$.

A partir de equações log-logísticas foram construídas as curvas de dose-resposta. Com base nos modelos ajustados, obtiveram-se as estimativas das doses de herbicida que proporcionariam $50 \%$ e $80 \%$ de controle ( $\mathrm{I}_{50} \mathrm{e}$ I80). Para efetuar os cálculos, optou-se pela inversão do modelo log-logístico em função de y, de acordo com Francischini et al. (2013):

$$
x=b * \sqrt{\frac{a}{y}}-1
$$

\section{Resultados e Discussão}

Os resultados obtidos dos tratamentos com o herbicida glyphosate, com os respectivos coeficientes da equação, valores de $\mathrm{I}_{50}$ e $\mathrm{I}_{80} \mathrm{e}$ fator de resistência (FR) sobre as plantas de $D$. insularis são apresentados na Tabela 1. As curvas de dose-resposta para este herbicida estão nas Figuras 1 e 2. De acordo com a fórmula proposta por Streibig (1988), valores de $b$, doses que proporcionam $50 \%$ de controle, muito baixos, demonstraram tendência à suscetibilidade da espécie aos herbicidas. $\mathrm{Na}$ área de Leópolis, o valor de "b" foi de 256,45, o que reflete a suscetibilidade das plantas de capim-amargoso. Para os demais locais, os valores foram sempre superiores a 1164,75.

Para o herbicida glyphosate não foi possível calcular os valores de $\mathrm{I}_{50}$ e $\mathrm{I}_{80}$ para biótipos de $D$. insularis provenientes de sementes da área de Cascavel, indicando alto grau de resistência ao glyphosate, $(\mathrm{FR}>10)$. Para as demais áreas testadas, com exceção de Leópolis (área suscetível), apesar dos valores de FR serem inferiores a 10, nenhuma dose testada proporcionou controle de $80 \%$, indicando a presença também de biótipos resistentes ao glyphosate. Segundo Rodrigues e Almeida (2011), as doses de glyphosate recomendadas para o controle de D.insularis variam de 720 a $1080 \mathrm{~g}$ i.a ha-1. 
Tabela 1. Estimativas dos parâmetros $a, b$ e $c$ e do coeficiente de determinação $\left(\mathrm{R}^{2}\right)$ do modelo log-logístico, ajustados para glyphosate e doses para $50 \%\left(\mathrm{I}_{50}\right)$ e $80 \%$ ( $\left.\mathrm{I}_{80}\right)$ de controle de D.insularis aos 28 D.A.A. Bandeirantes, PR -2013.

\begin{tabular}{lccccrrr}
\hline \multicolumn{1}{c}{ Local } & $a$ & \multicolumn{1}{c}{$b$} & $c$ & $\mathrm{R}^{2}$ & $\begin{array}{c}\mathrm{I}_{80} \\
\left(\mathrm{~g} \mathrm{e}^{2} \cdot \mathrm{ha}^{-1}\right)\end{array}$ & $\begin{array}{c}\mathrm{I}_{50} \\
\left(\mathrm{~g} \mathrm{e.a.} \mathrm{ha-1}^{-1}\right)\end{array}$ & \multicolumn{1}{c}{$\mathrm{FR}$} \\
\hline Cascavel & 36,01 & 3581,64 & $-0,6661$ & 0,79 & $>3840,00$ & $>3840,00$ & $>16,88$ \\
Palotina & 88,42 & 1612,87 & $-0,8368$ & 0,91 & $>3840,00$ & 1437,68 & 6,32 \\
S. Mariana & 92,24 & 1164,75 & $-0,6205$ & 0,85 & $>3840,00$ & 1424,90 & 6,26 \\
Maringá & 72,03 & 1389,42 & $-2,0463$ & 0,92 & $>3840,00$ & 2074,04 & 9,12 \\
Leópolis* & 100,49 & 256,45 & $-2,2805$ & 0,99 & 466,01 & 227,42 & 1,00 \\
\hline * Biótipo suscetível & & & & & & &
\end{tabular}

Em trabalhos realizados recentemente, alguns pesquisadores observaram falhas de controle de populações de D.insularis com o uso de glyphosate. Essas falhas foram relatadas em áreas em que o uso deste herbicida aumentou significativamente devido ao uso de organismos geneticamente modificados com a tecnologia Roundup Ready $\mathrm{RR}^{\circledR}$. Desenvolvimento de resistência ao glyphosate em D.insularis ocorreu no Estado de São Paulo em 2008, onde foram encontrados dois biótipos oriundos da mesma localidade, comprovadamente resistentes (Nicolai et al., 2010).

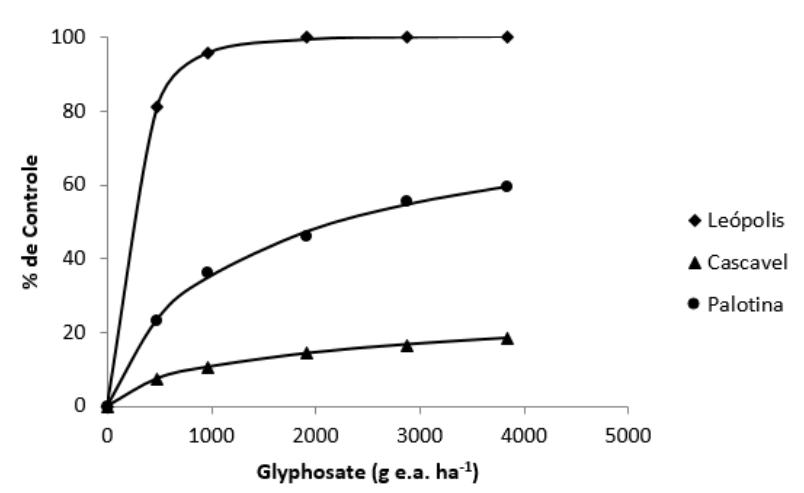

Figura 1. Curvas de dose resposta de biótipos de Digitaria insularis, coletados em áreas dos municípios de Leópolis, Cascavel e Palotina, para o herbicida glyphosate. Bandeirantes, PR 2013.

Nas curvas de dose-resposta de plantas de D. insularis provenientes de sementes coletadas nas áreas de Cascavel e Palotina (Figura 1), o controle com glyphosate foi menor que $20 \%$ e $60 \%$, respectivamente, com a aplicação da maior dose 3840 g i.a. ha ${ }^{-1}$, não alcançando $80 \%$ com nenhuma das doses testadas. Melo et al. (2012), avaliando o controle de Digitaria insularis resistente e suscetível ao glyphosate nos estádios de duas folhas (plantas mais novas) e de dois perfilhos (plantas mais velhas), observaram que as doses de glyphosate necessárias para controle satisfatório foram de 310,9 e $632,6 \mathrm{~g}$ i.a. ha ${ }^{-1}$ para plantas resistentes e de 175,5 e 474,1 g i.a. $\mathrm{ha}^{-1}$ para plantas suscetíveis, nos estádios de duas folhas e de dois perfilhos, respectivamente.

Nas curvas de dose-resposta de plantas de $D$. insularis oriundas de sementes coletadas nas áreas de Santa Mariana e Maringá (Figura 2), o controle com glyphosate foi menor que $60 \%$ e $63,5 \%$ com as doses de 2880 e 3840 g e.a. ha $^{-1}$ respectivamente. Isso evidencia que os biótipos ou populações de ambas as áreas são resistentes ao glyphosate, não atingindo $80 \%$ de controle, mesmo com o aumento de doses. Resistência de $D$. insularis ao glyphosate foi comprovada por Melo et al., (2012). Reinert et al., (2013), também confirmaram a ocorrência de resistência em populações de $D$. insularis nos estádios de 3 a 4 perfilhos com a aplicação de $7200 \mathrm{~g}$ e.a. ha ${ }^{-1}$ de glyphosate.

Os resultados do segundo experimento com o herbicida clethodim, visando ao controle de biótipos de $D$. insularis de áreas com problema de controle com o glyphosate, são apresentados na Tabela 2 e Figuras 3 e 4 . Segundo Gemelli et al. (2013), o herbicida clethodim está entre as melhores opções para o controle de capim-amargoso. 


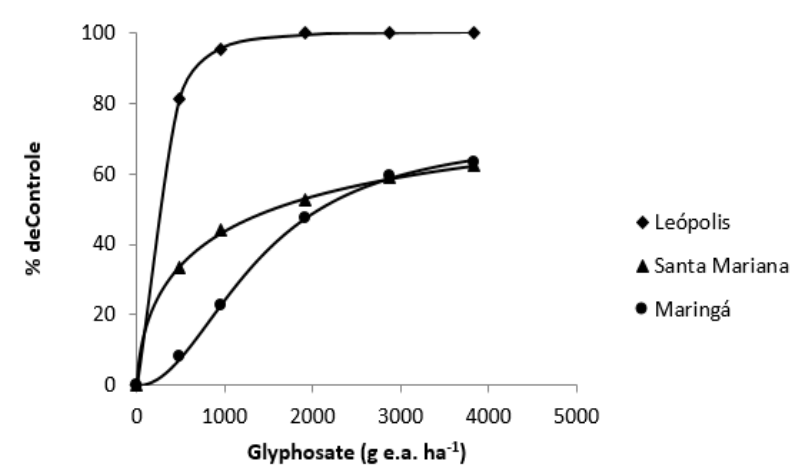

Figura 2. Curvas de dose-resposta de biótipos de Digitaria insularis, coletados em áreas dos municípios de Leópolis, Santa Mariana e Maringá, para o herbicida glyphosate. Bandeirantes, PR - 2013.
Os biótipos de $D$. insularis das áreas estudadas apresentaram variações na suscetibilidade ao herbicida clethodim. Para as regiões de Palotina e Maringá, as doses necessárias para o controle de $80 \%$ foram inferiores a $100 \mathrm{~g}$ i.a. ha ${ }^{-1}$. Resultados obtidos por Spader \& Matera, (2010) indicaram que o clethodim, em doses a partir de 96 g i.a. ha ${ }^{-1}$, foram eficazes no controle de plantas de Digitaria insularis tolerante ao glyphosate. Já, para as áreas de Santa Mariana e Cascavel os valores foram de 179,81 e 191,95 g i.a. ha ${ }^{-1}$, respectivamente, o que revela biótipos com sensibilidade diferencial ao clethodim.

Tabela 2. Estimativas dos parâmetros $a, b$ e $c$ e do coeficiente de determinação $\left(\mathrm{R}^{2}\right)$ do modelo log-logístico, ajustados para clethodim e doses para 50\% ( $\mathrm{I}_{50}$ ) e $80 \%$ ( $\mathrm{I}_{80}$ ) de controle de D.insularis aos 28 D.A.A. Bandeirantes, PR - 2013.

\begin{tabular}{|c|c|c|c|c|c|c|}
\hline Local & $\mathrm{a}$ & $\mathrm{b}$ & $\mathrm{c}$ & $\mathrm{R}^{2}$ & $\begin{array}{c}\mathrm{I}_{80} \\
\left(\mathrm{~g} \text { i.a. } \mathrm{ha}^{-1}\right)\end{array}$ & $\begin{array}{c}\mathrm{I}_{50} \\
\left(\text { g i.a. } \mathrm{ha}^{-1}\right)\end{array}$ \\
\hline Cascavel & 108,56 & 73,91 & $-1,0792$ & 0,98 & 191,95 & 63,84 \\
\hline Palotina & 97,72 & 58,93 & $-3,0536$ & 0,98 & 96,54 & 59,84 \\
\hline S. Mariana & 123,67 & 81,12 & $-0,7607$ & 0,97 & 179,81 & 48,74 \\
\hline Maringá & 97,93 & 51,36 & $-3,1849$ & 0,98 & 82,15 & 52,05 \\
\hline
\end{tabular}

Os resultados das curvas de doseresposta para o controle de D.insularis com o herbicida clethodim (Figura 3), de sementes coletadas na área de Cascavel, a dose de $96 \mathrm{~g}$ i.a. $\mathrm{ha}^{-1}$ proporcionou controle próximo a $65 \%$, demonstrando a possibilidade dos biótipos desta área serem mais tolerantes ao herbicida clethodim. Isso porque, a dose recomendada para controle de capim-amargoso é de $96 \mathrm{~g}$ i.a. $\mathrm{ha}^{-1}$, e tal controle foi obtido somente com $191,95 \mathrm{~g}$ i.a. $\mathrm{ha}^{-1}$. $\mathrm{Na}$ área de Palotina, apenas com a dose de $48 \mathrm{~g}$ i.a. ha ${ }^{-1}$ o controle das plantas de D.insularis oriundas de sementes coletadas foi abaixo de $80 \%$, as demais doses apresentaram controle acima de $80 \%$ mantendo a eficiência do herbicida clethodim.

Estudos realizados por Adegas et al. (2010), comprovaram que o controle de capimamargoso, quando realizado na fase inicial, apresenta níveis satisfatórios, aos sete dias após a aplicação (DAA) dos tratamentos. Com exceção do glyphosate, todos os herbicidas proporcionaram controle superior a $80 \%$, sendo os tratamentos mais eficientes o haloxyfopmethyl, o tepraloxydim e o paraquat. Ainda na avaliação de 14 DAA o grupo de tratamentos mais eficientes foram constituídos pelos herbicidas clethodim, clethodim + fenoxapropp-ethyl, haloxyfop-methyl, tepraloxydim e paraquat.

Os resultados obtidos com as sementes provenientes da região de Santa Mariana demonstram que a dose 96 g i.a. ha ${ }^{-1}$ controlou apenas $67 \%$, indicando também a possibilidade dos biótipos de plantas desta área serem mais tolerantes ao herbicida clethodim (Figura 4). Para as sementes coletadas na área de Maringá, apenas na dose $48 \mathrm{~g}$ i.a.ha ${ }^{-1}$ o controle de Digitaria insularis foi menor que $80 \%$, nas demais doses de clethodim o controle foi superior a $80 \%$. Considerando-se que no momento da aplicação as plantas se 
encontravam com três a quatro perfilhos, a expectativa era de que o herbicida clethodim apresentasse controle adequado em todas as áreas estudadas, o que não ocorreu com biótipos da área de Santa Mariana. Problema de controle de $D$. insularis com este herbicida também, foi observado por Melo et al. (2012).

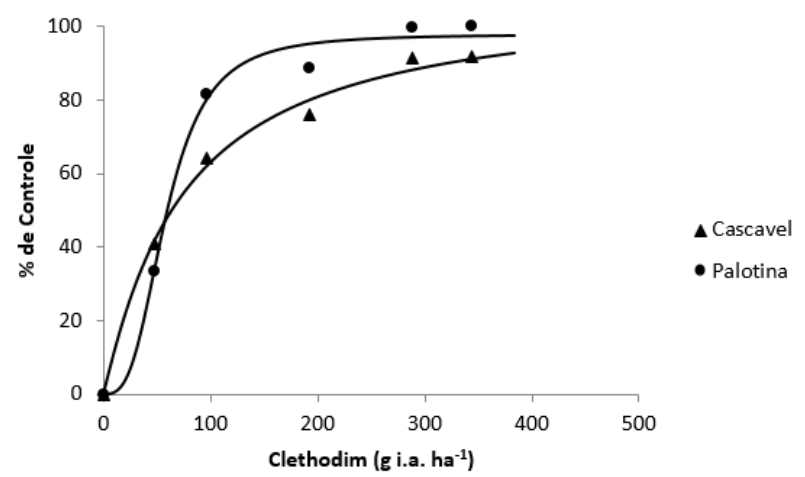

Figura 3. Curvas de dose-resposta de biótipos de Digitaria insularis, coletados em áreas dos municípios de Cascavel e Palotina, para o herbicida clethodim. Bandeirantes, PR - 2013.

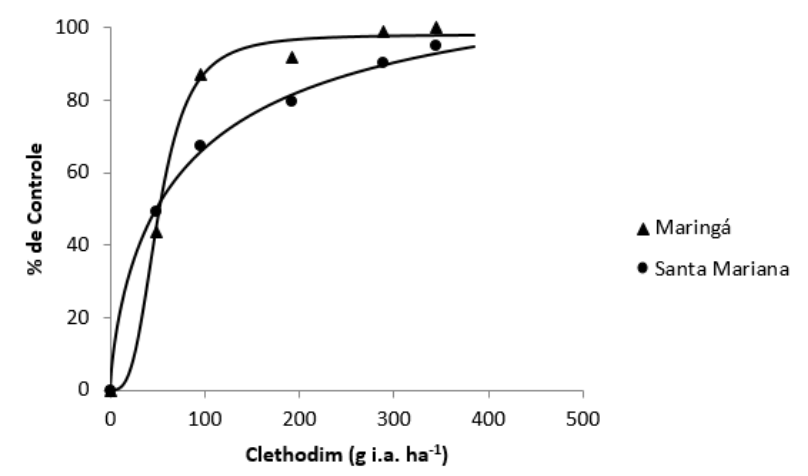

Figura 4. Curvas de dose-resposta de biótipos de Digitaria insularis, coletados em áreas dos municípios de Maringá e Santa Mariana, para o herbicida clethodim. Bandeirantes, PR - 2013.

Quando se tem biótipos de D.insularis resistentes na área, há dificuldade de controle, principalmente onde existem biótipos de resistência múltipla. A resistência múltipla ocorre quando um biótipo possui dois ou mais mecanismos de resistência distintos que conferem o comportamento resistente a um ou vários herbicidas com diferentes mecanismos de ação. Também pode ser considerado um caso de resistência múltipla quando o mecanismo de resistência é metabólico e que este metabolismo é comum a herbicidas de diferentes mecanismos de ação, e, portanto, neste caso a resistência é decorrente de apenas um mecanismo de resistência, porém este mecanismo confere múltipla resistência aos herbicidas de diferentes mecanismos de ação. Neste trabalho foram detectadas plantas de Digitaria insularis que não foram controladas pelas doses recomendadas de glyphosate e também de clethodim. No entanto, o aumento das doses de clethodim proporcionou níveis de controle adequado para as plantas de capim-amargoso nestes locais, o que reforça a ideia de que o ajuste de doses deste herbicida pode auxiliar no controle da espécie.

\section{Conclusões}

Os resultados permitiram concluir que no estado do Paraná já é frequente a ocorrência de biótipos de Digitaria insularis resistentes ao herbicida glyphosate, conforme mostrado nas áreas de Cascavel, Palotina, Maringá e Santa Mariana.

Além disso, nas áreas de Cascavel e Santa Mariana também foram encontrados biótipos de $D$. insularis que não foram controlados pelas doses recomendadas de clethodim, o que indica tolerância diferenciada dos biótipos de capim-amargoso a este herbicida e necessidade de uma adequação de manejo da comunidade infestante para evitar a ocorrência de resistência múltipla. Para Palotina e Maringá, o uso do clethodim nas doses recomendadas exerceu controle efetivo sobre a espécie.

\section{Referências}

Adegas, F.S. et al. Alternativas de controle químico de Digitaria insularis resistente ao herbicida glyphosate. In: CONGRESSO BRASILEIRO DA CIÊNCIA DAS PLANTAS DANINHAS, 27, 2010, Ribeirão Preto. Resumos... Ribeirão Preto: Sociedade 
Brasileira da Ciência das Plantas Daninhas, 2010, p.758-760.

Barros, A.A.M. et al. Interação entre herbicidas inibidores da ACCase e diferentes formulações de glyphosate no controle de capim-amargoso. Planta Daninha, v.32, n.3, p.619-627, 2014.

Brighenti, A.M; Oliveira, M.F. Biologia de plantas daninhas. In: OLIVEIRA JR., R.S.; CONSTANTIN, J.; INOUE, M.H. (eds.). Biologia e manejo de plantas daninhas. Curitiba: Omnipax, 2011. p.09.

Carvalho, L.B. et al. Detection of sourgrass (Digitaria insularis) biotypes resistant to glyphosate in Brazil. Weed Science, v.59, n.2, p.171-176, 2011.

Francischini, A. C et al. Curvas de dose-resposta e eficácia de herbicidas inibidores da enzima ALS aplicados em pré-emergência sobre espécies de Amaranthus. Revista Brasileira de Herbicidas, v.12, n.1, p.68-77, 2013.

Gemelli, A. et al. Estratégias para o controle de capim-amargoso (Digitaria insularis) resistente ao herbicida glyphosate na cultura milho safrinha. Revista Brasileira de Herbicidas, v.12, n.2, p.162-170, 2013.

Koger, C.H.; Reddy, K.N. Role of absorption and translocation in the mechanism of glyphosate resistance in horseweed (Conyza canadensis).Weed Science, v.53, n.1, p.84-89, 2005.

Machado, A.F.L. et al. Análise do crescimento de Digitaria insularis. Planta Daninha, v.24, n.4, p.641-647, 2006.

Machado, A.F.L. et al. Caracterização anatômica de folha, colmo e rizoma de Digitaria insularis. Planta Daninha, v.26, n.1, p.1-8, 2008.

Melo, M.S.C. et al. Alternativas para o controle químico de capim-amargoso (Digitaria insularis) resistente ao glyphosate. Revista Brasileira de Herbicidas, v.11, n.2, p.195-203, 2012.
Nicolai, M. et al. Monitoramento de infestações de populações de capim-amargoso (Digitaria insularis) suspeitas de resistência ao glifosato. In: CONGRESSO BRASILEIRO DA CIÊNCIA DAS PLANTAS DANINHAS, 27, 2010, Ribeirão Preto. Resumos... Ribeirão Preto: Sociedade Brasileira da Ciência das Plantas Daninhas, 2010. p. 943-946.

Reinert, C.S.; Prado, A.B.C.A.; Christoffoleti, P.J. Curvas de dose-resposta comparativas entre os biótipos resistente e suscetível de capimamargoso ao herbicida glyphosate. Revista Brasileira de Herbicidas, v.12, n.3, p.260-267, 2013.

Rodrigues, B.N.; Almeida, F.S. Guia de herbicidas. Londrina, PR: Edição dos autores, 6 Edição, 2011. 697 p.

Spader, V. et al. Manejo de azevém (Lolium multiflorum) resistente ao herbicida glyphosate.

In: CONGRESSO BRASILEIRO DA CIÊNCIA DAS PLANTAS DANINHAS, número, 26, 2008, Viçosa. Resumos... Viçosa: Sociedade Brasileira da Ciência das Plantas Daninhas/Embrapa Milho e Sorgo, 2008.

Spader, V.; Matera, J. Controle de capim amargoso tolerante e buva resistente ao herbicida glyphosate. In: CONGRESSO BRASILEIRO DA CIÊNCIA DAS PLANTAS DANINHAS, 27., 2010, Ribeirão Preto. Resumos... Ribeirão Preto: SBCPD, 2010.

Streibig, J.C. Herbicide bioassay. Weed Research, v.28, n.6, p.479-484, 1988. 